\title{
Nasal Suction System for Endoscopic Sinus Surgery
}

\author{
Preetam Chappity
}

\begin{abstract}
Introduction: Endoscopic sinus surgery has developed significantly in the past decade. The indications and the frequency of the surgery have increased tremendously. Performing this surgery in an awake patient under local anesthesia has numerous additional inherent problems as opposed to surgery under general anesthesia.
\end{abstract}

Technique: Our 'Continuous Suction Assembly' is a simple yet effective answer to these problems. It helps in providing a clear field during surgery. It also prevents aspiration and fogging and aids in accurate analys is of intraoperative blood loss.

Conclusion: Continuous suction technique is a cost-effective, simple technique to prevent many problems inherent to endoscopic sinus surgery under both local and general anesthesia. With proper preoperative counseling, the patient discomfort and cooperation can be improved drastically.

Keywords: Aspiration, Endoscopic sinus surgery, Nasal packing.

How to cite this article: Chappity P. Nasal Suction System for Endoscopic Sinus Surgery. Clin R hinol An Int] 2014;7(1):10-12.

\section{Source of support $\mathrm{Nil}$}

\section{Conflict of interest None}

\section{INTRODUCTION}

Endoscopic sinus surgery has progressed immensely in the past decade. The indications and so the frequency of its use has increased tremendously. In routine practice of an endoscopic surgeon, it is not unusual to have cases where bleeding is encountered in spite of good decongestion and surgical techniques. In such conditions, patient is definitely at a higher risk of aspirating blood, blood clots and packing material. A Iso, the surgeon has a bad surgical field which worsens the matter further. The aspiration of blood increases the morbidity in the postoperative period and sometimes obstruction of larynx and trachea with clots or dislocation of packing, sometimes lethal is not unheard of. Our technique is a very simple, cost-effective answ er to these problems which play an important role in the ultimate surgical outcome.

Assistant Professor

Department of ENT, All India Institute of Medical Sciences Bhubaneswar, Odisha, India

Corresponding Author: Preetam Chappity, Assistant Professor, Department of ENT, All India Institute of Medical Sciences, Bhubaneswar, Odisha, India, Phone: 9438884166 e-mail: drcpreetam@aiimsbhubaneswar.edu.in

\section{TECHNIQUE}

Preoperatively, the patient is counseled regarding the nasal airflow blockage and transient sense of fullness or discomfort in nasopharynx. A fter positioning, the patient in a supine position with $30^{\circ}$ head end el evation and applying topical anesthesia. A large size Foleys (No. 18) catheter is placed through the contralateral nostril (nonoperative side) and through the ipsilateral side (operative side) with the help of an endoscope and a curved ball probe (Fig. 1A), nasopharyngeal end of the Foley's catheter is pulled in placed in the ipsilateral nasal cavity. The tip of the catheter is then guided in the inferior meatus hinging below the inferior turbinate so that the tip is out of the operating field (Fig. 1B). Balloon of the Foley's catheter is inflated with 10 to $15 \mathrm{ml}$ of air in order to occlude the nasopharyngeal ai rway (Fig. IC). The outer end of Foley's catheter (contralateral nasal cavity) is attached to a continuous suction machine (Fig. 2). During the surgery, the suction assembly continuously sucks the blood, clots and excess airflow to render a clear surgical field for the surgeon. In our series of patients in whom we have used this technique regularly, only two patients till date have not opted for the catheter to be placed in the nasopharynx due to the fear of discomfort in all other patients no difficulties were encountered.

\section{DISCUSSION}

Surgery under local anesthesia has numerous advantages, like:

- Procedure can be a day-care procedure with minimal cost.

- As the patient is awake, the surgeon is gentle and so there is less mucosal injury.

- Due to usage of local vasoconstrictors and endogenous release of epinephrine the bleeding is comparatively less.

- Can be done in patients not fit for surgery under general anesthesia.

- M onitoring of vision and detecting complications early and managing it immediately is a significant advantage. $B$ ut there are also some disadvantages ${ }^{1,2}$ above like, the patient is uncomfortable with awareness of surgery being done, limitations in doing an extensive procedure, need for controlled sedation all throughout the surgery by anesthetist and due to depressed reflexes there is a definite chance of aspiration of blood clots or nasal packs. With aspiration, there is a lot of morbidity starting with patient discomfort, increased reflux due to swallowing of blood and estimation 

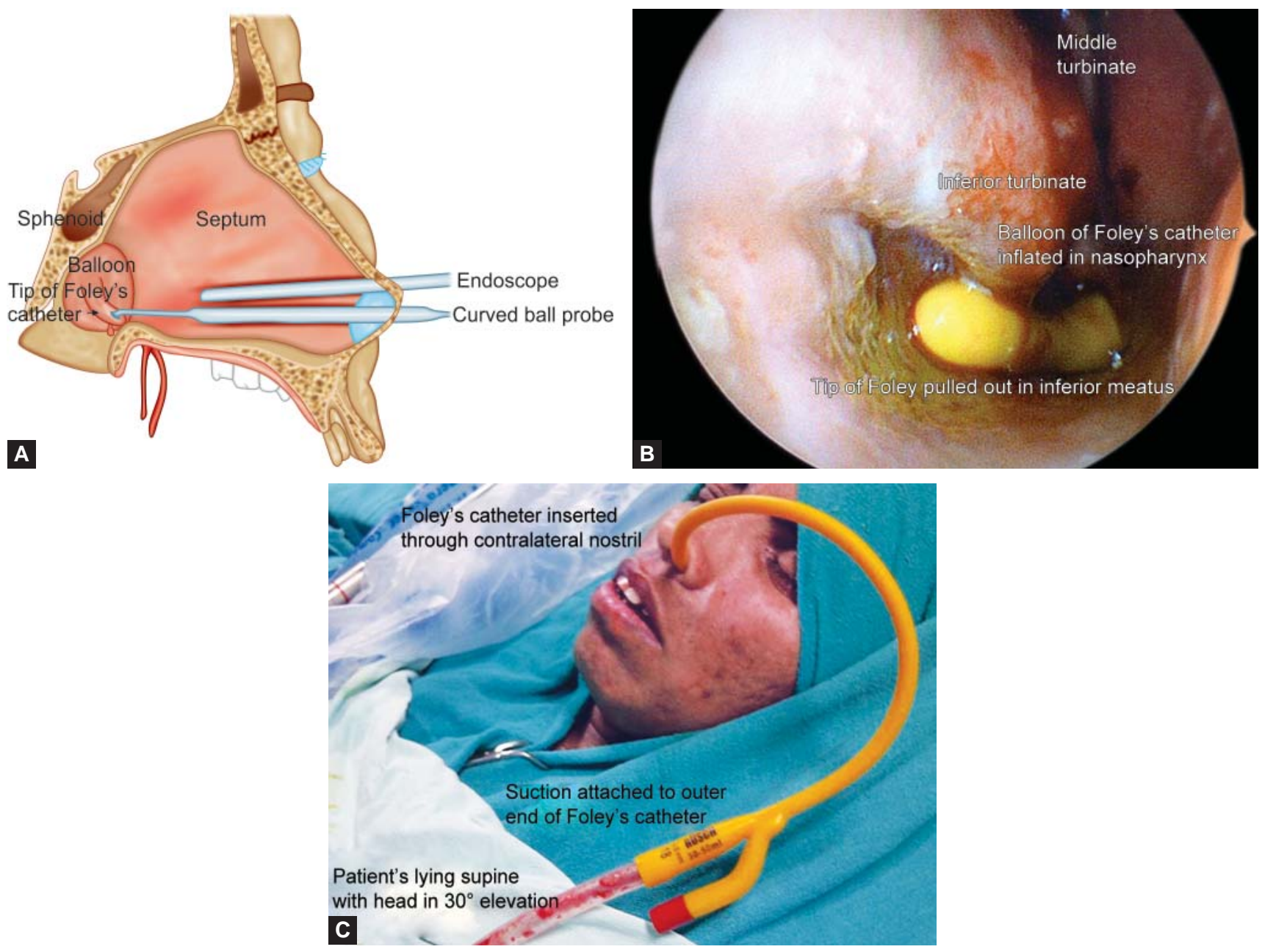

Figs 1A to C: The placement of the Foley's catheter for the suction system

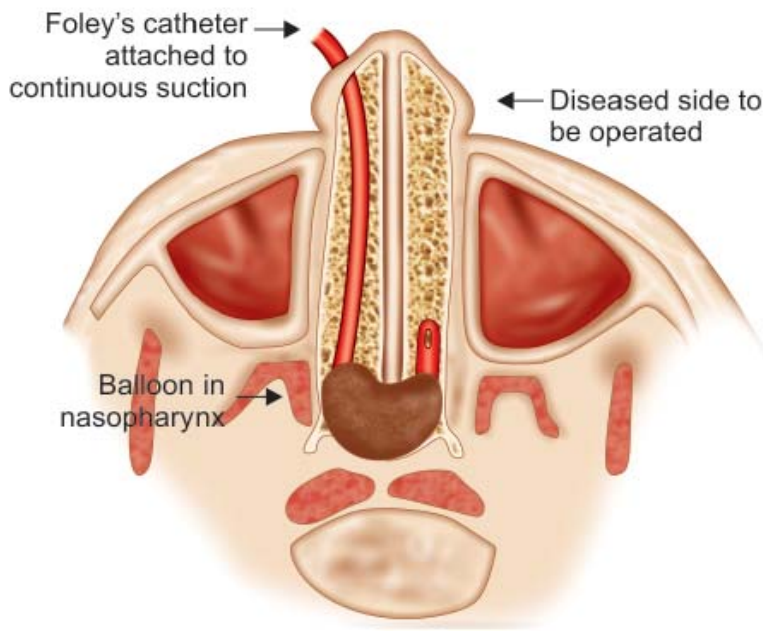

Fig. 2: Schematic diagram of cross-section at the inferior meatus

of blood loss is also hampered. Possibility of mortality is also a rare yet possible complication.

Our technique works very well if the patient is explained about the procedure and accepts the possible discomfort. In our experience, once the patient understands the inherent problems of the surgery and how this technique helps in decreasing those problems, there was no problem that we encountered. The associated minimal cost also helps, especially in developing countries like ours. The advantages in this technique are as follows:

1. Continuous atraumatic suction with a clear surgical field.

2. Avoids fogging on endoscope because of breathing during the surgery, by blocking both the posterior choanae with the inflated balloon of the catheter.

3. Avoidance of aspiration of blood or packs intraoperatively due to depressed reflexes.

4. A ccurate estimation of blood loss as none is swallowed or coughed out.

5. Use of bipolar cautery is easier as there is continuous suctioning of the smoke, with a clear surgical field.

6. Especially, useful in endoscopic drilling procedures like endoscopic dacrocystorhinostomy, where continuous saline irrigation is required.

Clinically, we had significant decrease in patient discomfort with better surgical ease. This technique enables us to operate more difficult pathologies and for prolonged time with accurate evaluation of blood loss. 


\section{CONCLUSION}

Continuous suction technique is a cost-effective, simple technique to prevent many problems inherent to endoscopic sinus surgery under local anesthesia. With good preoperative counseling, it helps immensely in improving surgical ease and decreasing patient discomfort.

\section{REFERENCES}

1. Weber R, Hochapfel F, L euwer R, Freigang B, D raf W. Tamponaden und Platzhalter in der endonasalen Chirurgie. HNO 2000;48:240-256.

2. Simmen $D$, Jones N. M anual of endoscopic sinus surgery: and its extended applications. Thieme Publication. N ew York. 2005.p.145. 